\title{
Horizons: Looking Back, Moving Forward
}

NaOmi Rutenberg, PhD

Deborah Weiss, MPH ${ }^{\mathrm{b}}$
The epidemic of human immunodeficiency virus (HIV) / acquired immunodeficiency syndrome (AIDS) continues to wreak havoc with lives and societies around the world. The good news is that significant progress in biomedical, behavioral, and epidemiological research has identified and described in detail the nature of HIV infection and its modes of transmission. We have learned much about which behaviors place people at risk and/or prevent them from seeking care. We also have some good evidence about what types of interventions work to prevent HIV transmission and increase the uptake and effectiveness of treatment. However, we know far less about how to roll out these interventions or their impact on preventing infections and extending life in the uncontrolled real world. There is also still much we do not know about providing HIV prevention, care, and treatment services to, for example, marginalized populations or about how to increase the use of both old and new prevention technologies-and there are new challenges being raised every month. Expanding our knowledge base in developing countries-where more than $90 \%$ of HIV-infected people live-is especially important as prevention, access, and adherence to treatment, as well as support of families and communities affected by HIV, are critically important for dealing with the pandemic.

It was within this context that the United States Agency for International Development (USAID) awarded a cooperative agreement in July 1997 to the Population Council (an international, nonprofit, nongovernmental organization [NGO]) for implementation of the operations research program, Global Leadership, Research, and Development-Best Practices in HIV/AIDS. From 1997 to 2008, this program—known as Horizons — was implemented by the Population Council, in close collaboration with the International Center for Research on Women, the Program for Appropriate Technology in Health, the International HIV/AIDS Alliance, Tulane University, Family Health International, and Johns Hopkins University. Spanning a crucial decade in the universal fight against HIV and AIDS, Horizons made major contributions to the identification and development of best practices associated with the prevention and mitigation of

aPopulation Council, Washington, DC

'Population Council, New York, NY

Address correspondence to: Naomi Rutenberg, PhD, Population Council, 4301 Connecticut Ave. NW, Ste. 280, Washington, DC 20008; tel. 202-237-9405; fax 202-237-8410; e-mail <nrutenberg@popcouncil.org >.

(c)2010 Association of Schools of Public Health 
HIV and AIDS in developing countries by conducting operations research to identify effective approaches for strengthening and scaling up of HIV prevention, care, and treatment programs.

Operations research is a problem-solving process comprising five steps: (1) problem identification and diagnosis, (2) strategy selection, (3) strategy experimentation and evaluation, (4) information dissemination, and (5) results utilization. Horizons focused on three broad priority areas of operations research: research on comprehensive approaches to HIV and AIDS prevention; research to improve the coverage, quality, and effectiveness of HIV and AIDS treatment; and research to expand efforts to mitigate effects of the epidemic on people infected and affected by HIV and AIDS. In other words, Horizons used an operations research approach to learn more about what types of interventions work to prevent HIV transmission, deliver care and support, and mitigate the impact of HIV on individuals, families, and their communities; how these interventions work; why they work; and what they cost.

Horizons did not have an explicit geographic focus within the developing world. Instead, it had a research agenda that guided the implementation of studies in one or more countries where the conditions for addressing priority questions and implementing research activities were favorable. This approach proved extremely effective in improving the quality and effectiveness of service-delivery programs in large part because it was applied research that places a premium on involving key stakeholders in the entire research process. These stakeholders included government ministries, local NGOs, people living with HIV and AIDS, research agencies, and international organizations, among others. The program collaborated with more than 380 groups in 28 countries, the majority of which were in Africa, followed by Asia and Latin America.

Four overarching objectives guided the implementation of the Horizons program:

1. Identify cutting-edge issues affecting the design and delivery of HIV and AIDS programs.

2. Suggest refinements for existing programs and activities.

3. Propose innovative approaches that could be tested through field-based, program-oriented operations research.

4. Recommend demonstrated best practices for implementation and going to scale.

While these objectives remained consistent, the program's specific research agenda evolved to respond to new developments in the pandemic and our under- standing of the determinants of infection and response At its outset, Horizons focused largely on issues related to HIV prevention for generalized epidemics and to medical care and nonmedical support for people living with HIV, including, for example, studies of the prevention and management of sexually transmitted infections-which increase vulnerability to HIV infection as much as tenfold-and involvement of people living with HIV and AIDS in the delivery of communitybased prevention and care services. Near Horizons' midpoint, the issue of treatment gained attention when significant price reductions for antiretroviral (ARV) drugs and increased availability of low-priced generic drugs offered the potential to significantly increase access to drug therapy for millions of infected people through large-scale treatment programs. Horizons, therefore, broadened its focus to examine critical questions such as the need for training of health-care workers, the difficulties surrounding patient adherence to complex drug regimens, and how the growing availability of ARVs might influence prevention efforts. Similarly, the advent of effective prevention of motherto-child transmission (PMTCT) therapies prompted Horizons to investigate the feasibility of integrating PMTCT services into antenatal care, use lessons learned to strengthen PMTCT programs, and address barriers to service delivery and uptake.

Horizons has had a tremendous reach over the past decade, conducting 146 research-related activities in 28 countries, including 21 global and seven regional projects. Equally important as the studies conducted under the Horizons program was the mandate to build research capacity and interest in research findings. As such, the methodology was synthesized into an operations research toolkit, which contains all of the necessary elements for designing an HIV-related operations study, including developing the research protocol, collecting and analyzing data, and turning research into practice. This toolkit is intended to allow other researchers to replicate the field-tested approaches developed and utilized by Horizons researchers.

There is little reason to conduct operations research unless the results are widely disseminated and discussed, and every effort is made to see that findings are utilized to improve policies and programs. With this objective in mind, we offer this special section of Public Health Reports, which contains six articles that provide a synopsis of some of the research conducted under the Horizons program from 1997 to 2008. Some of the research presented in these articles pertains to projects seeking to address HIV-related stigma and gender inequalities, and how they perpetuate HIV transmission. Other articles in this series examine 
Horizons programs that tackle concerns facing specific at-risk populations, such as men who have sex with men and their increased HIV risk, and orphans and vulnerable children. Finally, we provide a synthesis of studies that sought to improve the delivery of PMTCT services and ARV treatment in resource-limited settings.
We hope that in providing this backward-looking but forward-thinking review of work accomplished under the Horizons program, other organizations may find the results useful for their own program planning and implementation. 